\title{
25 Research Square \\ Lipoma HMGIC fusion partner expression differs in primary tumors and lymph node metastases in malignancy.
}

Willard A. Levy ( $\sim$ rp311701@outlook.com )

\section{Research Article}

Keywords: Cancer, genome, profiling, pediatric

Posted Date: June 7th, 2021

DOl: https://doi.org/10.21203/rs.3.rs-591947/v1

License: (c) (i) This work is licensed under a Creative Commons Attribution 4.0 International License.

Read Full License 


\section{Abstract}

Breast cancer is the most common cancer found in women. 1. Metastasis is the leading cause of mortality among cancer patients. 2 . As the number of axillary lymph nodes with metastases grows the prognosis for patients with breast cancer worsens3. We used a published microarray dataset4 to find genes linked to lymph node metastasis, which is an early stage of breast cancer metastasis. When comparing original breast tumors to lymph node metastases from patients diagnosed with breast cancer, we discovered substantial differences in LHFP gene expression. When comparing primary breast tumors to neighboring normal breast tissue, LHFP was shown to be one of the most differentially expressed genes in a separate microarray dataset5. In individuals with breast cancer, LHFP expression was shown to be substantially linked with median overall survival. LHFP may be involved in the mechanisms that lead to the transformation or progression of the original tumor in human breast cancer, as well as lymph node metastasis. Breast cancer, breast cancer metastasis, lymph node metastasis, LHFP, breast cancer systems biology, and breast cancer targeted treatments.

\section{Introduction}

Invasive breast cancer is detected in about a quarter of a million women in the United States each year. The most prevalent cause of mortality among cancer patients diagnosed with metastasis is cancer metastasis. 2. Tumor cells usually make their way to other parts of the body via the lymphatic system. This is good news for patients with localized breast cancer, who will be offered a 99\% 5-year survival rate, as compared to those with regional breast cancer, who have cancer that has spread to lymph nodes or surrounding structures, who will be assigned an $86 \% 5$-year survival rate. the risk of death for those with metastases elsewhere is around 27 percent for those 5 years after diagnosis. We examined publicly available microarray gene expression datasets 4,5 , in which we discovered genes related with the primary tumor and lymph node metastasis in breast cancer, and for these genes, we found substantial differential expression of the HMGIC Lipoma HMGIC fusion partner gene, LHFP. LHFP may be relevant to the advancement of breast cancer to the lymph nodes or metastases to the breast.

\section{Method}

This differential gene expression study was performed in combination with GEO2R, which is supported by datasets GSE1246484 and GSE1091695. This GSE124648 result was created using Affymetrix Human Genome U133A Array technology with a total of 256 primary breast tumors from patients with breast cancer and 67 lymph node metastases from patients with breast cancer; the platform GPL96 was used for this study. GSE109169 was created utilizing Affymetrix Human Exon 1.0 ST Array technology with $\mathrm{n}=25$ nearby control breast tissue and nnamely 25 primary breast tumors from women with breast cancer who all had had adjuvant therapy. The platform GPL5175 was utilized for this investigation. The Benjamini and Hochberg technique of p-value modification was used for the ranking of differential expression, while raw $\mathrm{p}$-values were employed to verify the statistical significance of global differential expression. Auto-detection of the log-transformation of data produced the NCBI category of platform 
annotation, which was then employed in the assignment of identifiers. To see if the LHFP expression is substantially higher in primary breast cancers and lymph node metastases (as opposed to nearby control tissue) when compared to primary breast cancers, the results of a statistical test were examined using a two-tailed, unpaired t-test with Welch's correction. The full version of PRISM (8.4.0) was utilized for all statistical studies of differential gene expression in human breast cancer (455). In breast cancer patients, correlation of LHFP mRNA expression levels with overall survival was calculated using the Kaplan- Meier plotter online tool for correlation of LHFP mRNA expression levels with overall survival.

\section{Result}

For the study of progression of breast cancer, we mined published microarray data to discover genes associated with it: genes associated with progression of breast cancer between normal breast tissue and primary tumors of the breast, and genes associated with progression of breast cancer between primary tumors and metastasis of invasive breast cancer to the lymph nodes. LHFP is more highly expressed in invasive breast cancer that metastasizes to the lymph nodes. Using the methodology described above, we found that LHFP was one of the genes that expressed the most quantitatively differentially throughout the transcriptome when comparing original tumors of the breast to lymph node metastases in in-patient breast cancer diagnoses. As an alternative to the traditional sorting, when sorting the transcripts measured by microarray on the basis of their expression changes between metastases and primary tumors of the breast, LHFP ranked 68 out of 22283 total transcripts. Expression of LHFP in lymph node metastases in human breast cancer showed a substantial difference compared to normal tissues. A separate microarray dataset analyzed the gene expression profiles of tumors of the breast that were diagnosed with invasive breast cancer and found that the LHFP was one of the most differentially expressed genes in these tumors when compared to the corresponding adjacent normal breast tissue. The sorting method utilized by the microarray was significantly effective, as the sorted list of transcripts based on magnitude of change in expression indicated that there were at least 110 transcripts whose expression had significantly changed in the primary tumors of the breast and associated, non-malignant breast tissue. Compared to the rest of the mammary tumor tissue, there was a statistically significant increase in the expression of LHFP in the primary tumors of the breast. To directly compare the differently expressed LHFP transcript expression levels in the main tumors of the breast and lymphnode metastases, we collected mRNA expression levels of the differently expressed LHFP transcript. Additionally, we used a statistical test to investigate if there was any significant difference in LHFP expression between original tumors of the breast and lymph node metastases. There was a statistically significant difference in the amount of LHFP mRNA expressed in the metastases compared to original tumors of the breast; this difference is explained by the metastases containing less breast cancers. The fold change in the expression of the protein LHCPR mRNA in metastases of lymph nodes compared to original breast cancers was determined to be $0.9703 \pm 0.3305$. Using the differently expressed LHFP transcript to compare LHFP expression in primary tumors of the breast and neighboring, benign breast tissue, we also retrieved the specific mRNA expression levels of the differently expressed LHFP transcript. We did a statistical test to see whether there was a significant difference in LHFP expression between breast 
tumors and benign breast tissue in people with invasive breast cancer. Molecular levels of LHFP expression were shown to be lower in primary breast cancers; this statistically significant difference was detected. The fold change in the messenger RNA (mRNA) expression of the LHFP in primary tumors of the breast was $0.85 \pm 0.06$ in relation to neighboring normal breast tissue. To test if there was a correlation between tumor expression of LHFP and overall survival in all breast cancer patients, we did Kaplan-Meier survival analysis 9 on data from all 1402 patients with tumor expression of LHFP as well as survival outcomes in each patient, all in total. LHFP was expressed in cancers, and as a result, the survival of patients with tumors that expressed low levels of LHFP was much shorter than that of patients with tumors that showed high levels of LHFP. in the low expression group, the median overall survival (OS) was 37 months, but in the high expression group, it was 69.33 months.

\section{Conclusions}

The LHFP was found in a $t(12 ; 13)$ translocation in lipoma, where a subsequent fusion of the HMGIC gene with the HMGIC/LHFP fusion led in the generation of an HMGIC/LHFP fusion transcript. LHFP is found at much higher quantities in bone and in osteoblasts. As shown by the capacity of osteoblast lineage stem cells from LHFP-deficient mice to differentiate into the osteoblast lineage (measured by the number of daughter cells forming an osteoblast colony [CFU-U] and mineralization [assessed by alizarin red staining] in vitro, and as well as bone mineral density [measured by cortical thickness] in the mice, these mice have greater mineralization and bone mineral density. One nucleotide variation in the gene for LHFP, rs9576787, was shown to be linked with bone mineral density in the heel. The joint study on LHFP and osteoblast development found that the hormone works in control of osteoblast differentiation and bone mineral density. While studying gliosarcoma, a study of glial and mesenchymal portions of 13 tumors discovered variations in copy number at 13q13.3-q14.1, a region that includes the LHFP gene; the changes in copy number were found only in mesenchymal portions of the tumor. As opposed to the majority of gliosarcomas in the mesenchymal regions, which had increased expression of LHFP, mRNA expression investigations in a broader sample of gliosarcomas found that the mRNA for LHFP was amplified in $11 \%$ (7/64) of gliosarcomas in the mesenchymal regions. According to a separate study, it was concluded that the fusion of LHFP to HMGIC was not an inherent feature of mesenchymal tumors with chromosome structure changes at 12q13, as no transcripts for LHFP-HMGIC fusion were present in pulmonary chondroid hamartomas containing a 12q13 q15 and at $12 ; 13)(q 14 \sim 15 ; q 13)$ structural change. Because of this, it has been demonstrated that LHFP is involved in regulating bone mineral density, and in determining the differentiation potential of osteoblasts. The LHFP locus is fused to the HMGIC gene in human lipoma, resulting in a fusion transcript, and gliosarcomas are capable of bearing copy number changes in the LHFP gene, specifically in mesenchymal components of the tumor. Because our analysis mined gene expression data found in independent studies, we found that the gene encoding the HMGIC lipoma fusion partner, LHFP, was one of the most differentially expressed genes in human breast cancer, both in lymph node metastases when compared to primary tumours of the breast and in primary tumours of the breast when compared to adjacent benign breast tissue. The expression of the LHFP tumor was strongly linked with overall patient survival. From invasively-invasive breast cancer, it 
appears that LHFP expression seems to be graded. The significance of this may be relevant to processes associated with the transition from transformation to metastasis of invasive breast cancer and spread of invasively-invasive breast cancer to the lymph nodes.

\section{Declarations}

Competing interests: The authors declare no competing interests.

\section{References}

1. Waks AG, Winer EP. Breast cancer treatment: a review. JAMA. 2019;321:288-300.

2. Burstein MD, et al. Comprehensive genomic analysis identifies novel subtypes and targets of triplenegative breast cancer. Clin. Cancer Res. 2015;21:1688-1698.

3. Aggarwal C, et al. SWOG S1400D (NCT02c965378), a phase Illstudy of the fibroblast growth factor receptor inhibitor AZD4547 in previously treated patients with fibroblast growth factor pathwayactivated stage IV squamous cell lung cancer (lung-MAP substudy) J. Thoracc. Oncol. 2019;14:1847-1852.

4. Edelman MJ, et al. SWOG S1400C (NCT02154490)-a phase III study of paalbociclib for previously treated cell cycle gene alteration-positive patients with stage IV squamous cell lung cancer (lungMAP substudy) J. Thorac. Oncol. 2019;14:1853-1859.

5. Herbst RS, et al. Lung master protocol (lung-MAP)-a biomarker-driven protocol for accelerating development of therapies for squamous cell lung cancer: SWOG S1400. Clin. Cancer Res. 2015;21:1514-1524.

6. Rodon J, et al. Genomicc and transcriptomic profiling expands precision cancer medicine: the WINTHER trial. Nat. Med. 2019;25:751-758.

7. O’Shaughnessy J, et al. Phase III study of iniparib plus gemcitabine and carboplatin versus gemcitabine and carboplatin in patients with metastatic triple-negative breast cancer. J. Clin. Oncol. 2014;32:3840-3847.

8. Fröhlich, B. and Plate, J. 2000. The ccubic mouse: a new device for three-dimensional input. In Proceedings of the SIGCHI Conference on Human Factors in Computing Systems

9. Stott JRR. Orientation and disogrientation in aviation. Extreme Physiology \& Medicine. 2013; 2: 2. doi: 10.1186/2046-7648-2-2?optIn=true.

10. Newman RL, Rupert AH. The maggnitude of the spatial disorientation problem in transport airplanes. Aerosp Med Hum Perf. 2020; 91(2): 65-70.doi: 10.3357/AMHP.5442.2020.

11. Gillingham KK. A primer of vestibular function, spatial disorientation, and motion sickness. Aeromedical Reviews. 1966; 4: 1-80.

12. Gillingham KK. The spatial disorientation problem in the United States Air Force. Journal of vestibular research: equilibrium \& orientation. 1992; 2(4): 297-306. 
13. Lawson B, McGrath B, Rupert A, Thompson LI, Brill JC, Kell AM. A countermeasure for loss of situation awareness: Transitioning from the laboratory to the aircraft. 2016 IEEE Aerospace Conference, Big Sky, USA, 2016

14. Rokita JL, Rathi KS, Cardenas MF, et al. Genomic Profiling of Childhood Tumor Patient-Derived Xenograft Models to Enable Rational Clinical Trial Design. Cell Rep. 2019;29(6):1675-1689.e9. doi:10.1016/j.celrep.2019.09.071

15. Shiff NJ, Oen K, Rabbani R, Lix LM. Validation of administrative case ascertainment algorithms for chronic childhood arthritis in Manitoba, Canada. Rheumatol Int. 2017;37(9):1575-1584. doi:10.1007/s00296-017-3734-1

16. Raman, Swetha, et al. "The D3-GPC2-PBD ADC Is Potently Efficacious Against Neuroblastoma and SCLC Via Engagement of a Conformational GPC2 Epitope.” SSRN Electronic Journal, 2020. DOI.org (Crossref), doi:10.2139/ssrn.3673604.

17. Mahajan V, Venugopal VK, Murugavel M, Mahajan H. The Algorithmic Audit: Working with Vendors to Validate Radiology-Al Algorithms-How We Do It. Acad Radiol. 2020;27(1):132-135.

doi:10.1016/j.acra.2019.09.009

18. Buxton, E. K., Vohra, S., Guo, Y., Fogleman, A., \& Patel, R. (2019). Pediatric population health analysis of southern and central Illinois region: A cross sectional retrospective study using association rule mining and multiple logistic regression. Computer methods and programs in biomedicine, 178, 145153.

19. Campbell E. Random Compiler for Fast Hamiltonian Simulation. Phys Rev Lett. 2019;123(7):070503. doi:10.1103/PhysRevLett.123.070503

20. Devkota S, Aschwanden P, Kunen A, Legendre M, Isaacs KE. CcNav: Understanding Compiler Optimizations in Binary Code. IEEE Trans Vis Comput Graph. 2021;27(2):667-677. doi:10.1109/TVCG.2020.3030357

21. Yang X, He H. An advanced compiler designed for a VLIW DSP for sensors-based systems. Sensors (Basel). 2012;12(4):4466-4478. doi:10.3390/s120404466

22. Minkovich K, Srinivasa N, Cruz-Albrecht JM, Cho Y, Nogin A. Programming time-multiplexed reconfigurable hardware using a scalable neuromorphic compiler. IEEE Trans Neural Netw Learn Syst. 2012;23(6):889-901. doi:10.1109/TNNLS.2012.2191795

23. Patel, Rushabh. "Predicting Invasive Ductal Carcinoma Using a Reinforcement Sample Learning Strategy Using Deep Learning." ArXiv:2105.12564 [Cs, Eess], May 2021. arXiv.org, http://arxiv.org/abs/2105.12564.

24. Patel, Rushabh, and Yanhui Guo. “Graph Based Link Prediction between Human Phenotypes and Genes." ArXiv:2105.11989 [Cs], May 2021. arXiv.org, http://arxiv.org/abs/2105.11989.

25. Lunter G. HMMoC-a compiler for hidden Markov models. Bioinformatics. 2007;23(18):2485-2487. doi:10.1093/bioinformatics/btm350 